\title{
openheart Non-steroidal anti-inflammatory drug use in acute myopericarditis: 12-month clinical follow-up
}

\author{
Jan Berg, ${ }^{\oplus 1}$ Marina Lovrinovic, ${ }^{1}$ Nora Baltensperger,${ }^{1}$ Christine K Kissel, ${ }^{1}$ \\ Jan Kottwitz, ${ }^{1}$ Robert Manka, ${ }^{1}$ Dimitri Patriki, ${ }^{1}$ Frank Scherff, ${ }^{1}$ Christian Schmied, ${ }^{1}$ \\ Ulf Landmesser, ${ }^{2}$ Thomas F Lüscher, ${ }^{3,4}$ Bettina Heidecker ${ }^{\oplus 1,2}$
}

To cite: Berg J, Lovrinovic M, Baltensperger N, et al. Nonsteroidal anti-inflammatory drug use in acute myopericarditis: 12-month clinical follow-up. Open Heart 2019;6:e000990. doi:10.1136/ openhrt-2018-000990

$\mathrm{JB}$ and $\mathrm{ML}$ contributed equally.

Received 10 December 2018 Revised 20 February 2019 Accepted 4 March 2019
Check for updates

\section{(c) Author(s) (or their} employer(s)) 2019. Re-use permitted under CC BY-NC. No commercial re-use. See rights and permissions. Published by BMJ.

${ }^{1}$ Cardiology, University Heart Center, Zurich, Switzerland ${ }^{2}$ Charité Universitätsmedizin, Campus Benjamin Franklin, Berlin, Germany

${ }^{3}$ Center for Molecular Cardiology, University of Zurich, Zurich, Switzerland

${ }^{4}$ Royal Brompton and Harefield Hospitals and Imperial College, London, UK

\section{Correspondence to}

Dr Bettina Heidecker; bettina. heidecker@gmail.com

\section{ABSTRACT}

Objective Clinical data on the effect of non-steroidal anti-inflammatory drugs (NSAIDs) in myopericarditis are limited. Since NSAIDs are standard therapy in pericarditis, we retrospectively investigated their safety in myopericarditis.

Methods In a retrospective case-control study, we identified 60 patients with myopericarditis from September 2010 to August 2017. Diagnosis was based on clinical criteria, elevated high-sensitivity troponin T and cardiac magnetic resonance imaging (CMR). All patients received standard heart failure therapy if indicated. Twenty-nine patients $(62 \%)$ received NSAIDs (acetylsalicylic acid: $n=7$, average daily dose $=1300 \mathrm{mg}$ or ibuprofen: $n=22$, average daily dose $=1500 \mathrm{mg}$ ) for an average duration of 4 weeks. To create two cohorts with similar baseline conditions, 15 patients were excluded. Three months after diagnosis, 29 patients were re-evaluated by CMR to measure late gadolinium enhancement (LGE).

Results Baseline characteristics of those treated with or without NSAIDs were similar. Mean age was $34( \pm 13)$ years, $6(13 \%)$ were women. Mean left ventricular ejection fraction (LVEF) was $56 \%( \pm 5) .82 \%$ of the patients (14 of 17) treated with NSAIDs experienced a decrease in LGE at 3 months, while it was only $58 \%$ (7 of 12) of those without NSAIDs $(p=0.15)$. At 12-month follow-up, one of the patients treated without NSAIDs experienced polymorphic ventricular tachycardia (VT) with cardiac arrest, while one of the patients with NSAIDs experienced non-sustained VT. Conclusions This is the first case-control study demonstrating that NSAIDs are safe in patients with myopericarditis and preserved LVEF. Our data suggest that this drug class should be tested prospectively in a large randomised clinical trial.

\section{INTRODUCTION}

The treatment of myocarditis remains a clinical challenge. ${ }^{1-3}$ Current position statements recommend standard heart failure therapy and symptomatic treatment in haemodynamically stable patients with myocarditis. ${ }^{4}$ Thus, the first-line medication is standard heart failure therapy in those with markedly depressed left ventricular function. ${ }^{5} 6$ In patients with aggressive

\section{Key questions}

What is already known about this subject?

- Recommendations about the use of non-steroidal anti-inflammatory drugs (NSAIDs) in myopericarditis have been controversial in the literature. Since pericarditis requires anti-inflammatory treatment to prevent severe complications such as constrictive pericarditis, the involvement of the myocardium has often caused a clinical dilemma, as the recommendation for myocarditis is to avoid NSAIDs.

What does this study add?

- Our retrospective case-control study shows that high-dose ibuprofen and aspirin appear to be safe in the setting of acute myopericarditis with normal left ventricular ejection fraction (LVEF). A decrease in late gadolinium enhancement (LGE) on cardiac magnetic resonance imaging after 3 months was more frequent in those patients treated with NSAIDs as compared with those not treated; however, the difference did not reach level of significance.

How might this impact on clinical practice?

- NSAIDs are a valid and safe treatment option in the setting of acute myopericarditis. This finding is of high clinical relevance as prior mouse studies suggested this drug class to be associated with a higher mortality in myocarditis. Our study is the first case-control study in humans to assess the effect of NSAIDs in myocarditis on top of standard heart failure therapy. We suggest that NSAIDs should be tested prospectively for efficacy in a large randomised clinical trial.

autoimmune myocarditis, such as giant cell myocarditis or eosinophilic necrotising myocarditis, a combination of high-dose steroids and cyclosporine is recommended, sometimes in combination with muronomab CD3 ${ }^{7-9}$ Non-steroidal anti-inflammatory drug (NSAID) therapy has been a cornerstone of the symptomatic therapy of uncomplicated pericarditis. ${ }^{10}$ However, experimental studies in murine models suggested that NSAIDs may deteriorate the clinical course 
of myocarditis. ${ }^{11-14}$ Similarly, deleterious effects such as worsening inflammation and cell necrosis, ${ }^{11}$ increased mortality, ${ }^{12}$ viral titres ${ }^{13}$ and focal ventricular thinning ${ }^{14}$ were reported in the 1980s and 1990s. Also, NSAIDs have been shown to increase blood pressure ${ }^{15}$ and may impair renal function. ${ }^{16}$

Recently, cardiac magnetic resonance imaging (CMR) has evolved as the gold standard of non-invasive diagnostic imaging in haemodynamically stable patients with myocarditis. ${ }^{17} 18$ Signs of disease activity can be found in the myocardial and pericardial layer or both leading to the term myopericarditis. Patients with myopericarditis frequently experience chest pain, ${ }^{19}$ which can be challenging to treat. Drugs with limited anti-inflammatory effects such as acetaminophen may have inadequate analgesic function, increase blood pressure ${ }^{20}$ and impair renal function ${ }^{21}$-effects that may further deteriorate left ventricular performance. To our knowledge, there have been two studies examining the effects of NSAIDs in myopericarditis. ${ }^{22}{ }^{23}$ In the first study, Buiatti and colleagues investigated the management and outcome of 62 patients with myopericarditis who were treated with NSAIDs. ${ }^{22}$ Diagnosis of pericarditis was based on clinical presentation (chest pain, influenza-like symptoms weeks prior, pericardial friction rub). Myocardial involvement was defined by elevated troponin I levels or wall motion abnormalities on echocardiography. Out of 62 patients, $61(98 \%)$ received NSAIDs as first choice. During a minimal follow-up of 1 year, there were no events of death, new onset heart failure or wall motion abnormalities on echocardiogram. ${ }^{22}$

The second study was a multicentre study using CMR in addition to standard clinical tests for the diagnosis of myopericarditis in 140 patients of an overall population of 486 cases with pericarditis with and without myocardial involvement. There were 106 patients with myocardial involvement receiving NSAID therapy. Median follow-up was 36 months with no cases of death or heart failure being reported..$^{23} \mathrm{y}$

In summary, there have been only two clinical studies investigating outcomes of patients with myopericarditis receiving NSAID therapy. ${ }^{22}{ }^{23}$ Both studies suggested that this drug class could be used safely in this patient population. However, NSAIDs continue to be a grey area in the field of myopericarditis. In that regard, guidelines for myocarditis recommend to avoid this drug class, ${ }^{4}$ while guidelines for pericarditis with myocardial involvement suggest that data for NSAID use are controversial. ${ }^{10}$ Since insufficient treatment of pericarditis can be detrimental and lead to irreversible complications, there is a great need to treat the pericardial component of myopericarditis. ${ }^{24} 25$

To the best of our knowledgye, there is no literature comparing the effects of NSAIDs with standard heart failure therapy in myopericarditis and no study evaluated the effect of NSAIDs on the dynamics of late gadolinium enhancement (LGE) in myopericarditis-a major risk marker for adverse cardiovascular events. ${ }^{26-28}$
In this work, we sought to address both of these issues by performing a retrospective case-control study in patients with myopericarditis to evaluate (1) if the use of NSAIDs is safe as compared with standard heart failure therapy and (2) if NSAIDs affect the time course of LGE on CMR 3 months post diagnosis.

\section{METHODS}

\section{Patients and controls}

We performed a retrospective case-control study of patients who have been diagnosed with myopericarditis at the University Hospital Zurich between September 2010 and August 2017. Myopericarditis was diagnosed in 60 patients based on clinical presentation, elevated cardiac enzymes, ECG and CMR after the exclusion of obstructive coronary artery disease by coronary angiography by cardiac catheterisation or CT. ${ }^{19}{ }^{29}$ To create a homogenous cohort that only reflects acute myopericarditis, the analysis was restricted to patients with recent symptom onset ( $\leq 10$ days).

In this selected group of patients with myopericarditis, we compared those who were treated with standard heart failure therapy versus those receiving NSAIDs in addition to standard heart failure therapy. The two groups were matched according to baseline criteria such as left ventricular ejection fraction (LVEF) and age. Standard heart failure therapy contained beta-blocking agents, ACE inhibitors, angiotensin II receptor blockers and diuretics. NSAIDs used in this study included acetylsalicylic acid (ASA) or ibuprofen. Patients using other types of NSAIDs such as selective cyclooxygenase-2 inhibitors or steroids were excluded to reduce possible confounding factors by creating homogenous groups. Additional exclusion criteria were malignant arrhythmias, other pre-existing cardiomyopathies and fulminant myocarditis. Evaluation of patients was performed with local ethics committee approval and written informed consent from all patients.

\section{Clinical follow-up}

Patients were scheduled for follow-up examination 3, 6 and 12 months post diagnosis of myopericarditis. Data from these visits were screened for major adverse cardiovascular events defined as worsening LVEF, malignant arrhythmias (eg, ventricular tachycardia or fibrillation), worsening of LGE on CMR, all-cause mortality and cardiovascular death. Clinical history was obtained to screen for symptoms such as severe chest pain, dyspnea or palpitations. After a complete physical examination, routine blood tests were obtained including serum creatinine to screen for deterioration in renal function under NSAID therapy. Patients underwent clinical examination including echocardiography, 12-lead ECG, 48-hour Holter monitoring and exercise stress testing with bicycle and ramp protocol.

\section{CMR examination at baseline and at 3-month follow-up}

CMR examinations were performed on a 1.5 or 3.0 Tesla scanner (SiemensSkyra, Erlangen, Germany 


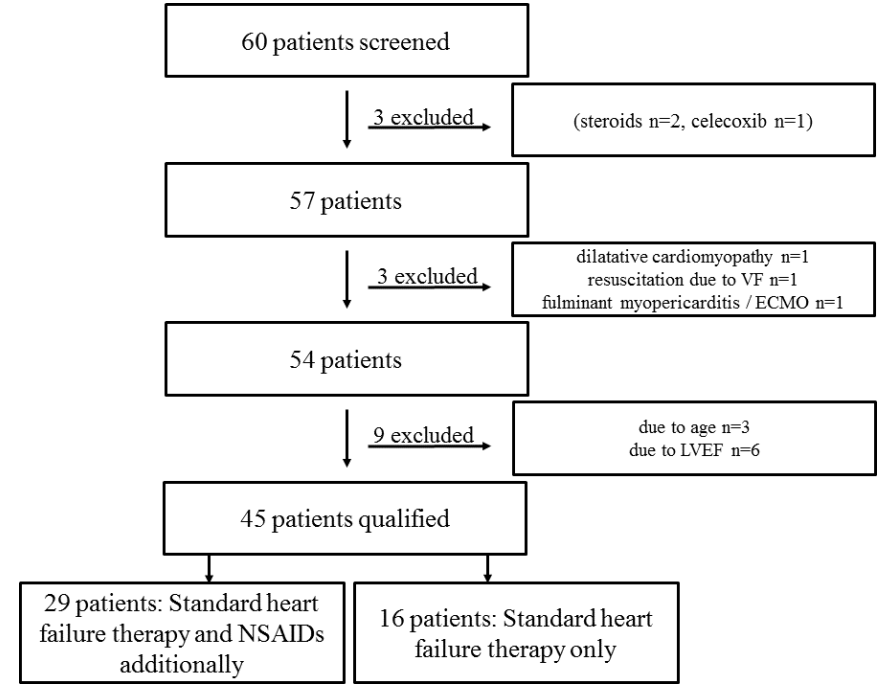

Figure 1 Patient enrolment. This flow chart shows the recruitment of patients and exclusion criteria. ECMO, extracorporeal membrane oxygenation; LVEF, left ventricular ejection fraction; NSAID, non-steroidal anti-inflammatory drug; VF, ventricular fibrillation.

or Philips Achieva, Best, The Netherlands) using an ECG-gated breath-hold protocol. Myopericarditis was diagnosed based on cine-CMR, T2-weighted imaging and T1-weighted LGE imaging as described previously. ${ }^{19} 29$ Ten minutes after intravenous administration of a gadolinium-based contrast agent, LGE short-axis images were generated. Routine CMR reporting included the evaluation of wall motion abnormalities and LVEF. Definition of pericardial involvement included pericardial thickening or effusion on CMR. Starting in 2016, our internal protocol also included follow-up CMR 3 months after diagnosis to identify LGE dynamic. The extent of LGE was evaluated in each slice and LGE dynamic from baseline to 3-month follow-up (increase, decrease or complete disappearance of LGE) was stated in the radiology report.

\section{Statistical analysis}

Data are presented as mean \pm SD. Numerical values were analysed with Wilcoxon rank sum test, and $\chi^{2}$ test was used for categorical values. Fisher's exact test was used to detect differences for sparse binary data in baseline conditions. A p value $<0.05$ was considered statistically significant. Analyses were performed with SPSS V.23. The first authors had full access to the data and take responsibility for the integrity of data analysis.

\section{RESULTS}

\section{Patient characteristics}

To reduce the risk for potential confounding factors, we excluded patients who received other anti-inflammatory medications such as steroids $(n=2)$ or celecoxib $(n=1)$. Three patients had to be excluded because they met exclusion criteria. Of those, one patient presented with pre-existing dilated cardiomyopathy. The second patient was hospitalised after resuscitation due to ventricular fibrillation and the third with cardiogenic shock requiring treatment with extracorporeal membrane oxygenation in the intensive care unit. After the initial enrolment, patients without NSAID therapy were older and had lower LVEF. In order to create two groups with similar baseline conditions, another six patients were excluded due to LVEF and three patients were excluded due to age. Details of patient enrolment are illustrated in figure 1 .

Forty-five patients with myopericarditis qualified for our retrospective case-control study. Patients treated with $(n=29)$ and without NSAIDs $(n=16)$ were by design similar at baseline (table 1). Importantly, there was no significant difference in age, gender, body mass index (BMI), tobacco use, cardiac enzymes and inflammatory parameters. Thirty-nine patients $(87 \%)$ were men and mean age was $34 \pm 13$ years. LVEF (calculated by MRI) was normal or mildly decreased $(>50 \%)$ in all patients treated with NSAIDs while it was normal in 13/16 patients (81\%) treated without NSAIDs. Three out of 16 patients treated without NSAIDs presented with mildly impaired LVEF. Mean LVEF in the overall patient population $(n=45)$ was $56 \% \pm 5 \%(57 \pm 4$ in the group treated with NSAIDs vs $55 \pm 6$ in the group without NSAIDs, $\mathrm{p}=0.09$ ). Eighteen patients were smokers $(40 \%)$ and mean BMI was $26 \pm 4$ $\mathrm{kg} / \mathrm{m}^{2}$. Mean high sensitivity troponin $\mathrm{T}$ was $757 \pm 1453$ $\mathrm{ng} / \mathrm{L}$, mean NT-pro-brain natriuretic peptide $635 \pm 1051$ $\mathrm{ng} / \mathrm{L}$ and mean myoglobin $82 \pm 85 \mu \mathrm{g} / \mathrm{L}$. Mean C reactive protein was $45 \pm 45 \mathrm{mg} / \mathrm{L}$ and mean leucocyte count $8.8 \pm 2.9 \mathrm{G} / \mathrm{L}$. Presenting symptoms at the time of diagnosis included chest pain in 42, dyspnea in 10 and syncope in 2 cases.

\section{Medication}

A total of 29 patients received ASA ( $\mathrm{n}=7$, mean daily dose $=1300 \mathrm{mg})$ or ibuprofen $(\mathrm{n}=22$, mean daily dose $=1500 \mathrm{mg}$ ) for an average duration of 4 weeks in addition to standard heart failure therapy. Five patients treated with NSAIDs received additional therapy with colchicine. The remaining 16 patients received standard heart failure therapy as indicated. Heart failure therapy included beta blockers $(n=9)$, ACE inhibitors $(n=18)$, angiotensin receptor blockers $(n=2)$ and diuretics $(n=1)$. None of the patients received aldosterone antagonists.

\section{Clinical follow-up at 3 months}

All patients were scheduled for clinical follow-up at 3 months. Follow-up data were obtained from 40 patients, while 5 were lost to follow-up ( 4 in the NSAID group and 1 in the standard heart failure group). Mean follow-up time was $12.1 \pm 9.6$ months (median 11 months, range 1-47 months). Eight patients (18\%) continued to report occasional brief episodes of chest pain at 3-month follow-up; six of them were in the NSAIDs group $(6 / 29$ patients $=21 \%$, ASA: $n=2$, ibuprofen: $n=4$ ), two of them in the standard heart failure therapy group (2/16 patients $=13 \%)$. One patient $(6 \%)$ in the standard heart failure therapy group reported episodes of dyspnea on 
Table 1 Baseline conditions of patients with and without non-steroidal anti-inflammatory drug (NSAID) therapy

\begin{tabular}{|c|c|c|c|}
\hline & NSAID (n=29) & No NSAID $(n=16)$ & $P$ value \\
\hline Mean age (SD) & $32(13)$ & $37(13)$ & 0.15 \\
\hline Male gender, $\mathrm{n}(\%)$ & $26(90)$ & $13(81)$ & 0.43 \\
\hline Mean LVEF on CMR (\%) & $57(4)$ & $55(6)$ & 0.09 \\
\hline Body mass index—mean, $\mathrm{kg} / \mathrm{m}^{2}$ (SD) & $26(4)$ & $27(4)$ & 0.5 \\
\hline Smoker, n (\%) & $12(41)$ & $6(38)$ & 1 \\
\hline CAD, n (\%) & $2(7)$ & 1 (6) & 1 \\
\hline Hypertension, n (\%) & $3(10)$ & $3(19)$ & 0.65 \\
\hline Dyslipidaemia, n (\%) & $3(10)$ & $3(19)$ & 0.65 \\
\hline Diabetes, n (\%) & 1 (3) & 0 & 1 \\
\hline Mean troponin (ng/L) & $922( \pm 1770)$ & $459( \pm 580)$ & 0.18 \\
\hline Mean NT-proBNP (ng/L) & $547( \pm 489)$ & $812( \pm 1744)$ & 0.48 \\
\hline Mean myoglobin ( $\mu \mathrm{g} / \mathrm{L})$ & $87( \pm 79)$ & $73( \pm 101)$ & 0.22 \\
\hline Mean CRP (mg/L) & $47( \pm 42)$ & $42( \pm 52)$ & 0.49 \\
\hline Mean leucocytes (G/L) & $8.9( \pm 2.5)$ & $8.6( \pm 3.6)$ & 0.45 \\
\hline Mean creatinine $(\mu \mathrm{mol} / \mathrm{L})$ & $76( \pm 11)$ & $77( \pm 15)$ & 0.59 \\
\hline \multicolumn{4}{|l|}{ Medications on admission } \\
\hline Betablocker, n (\%) & 0 & $1(6)$ & 0.36 \\
\hline ACE inhibitor, n (\%) & $3(10)$ & 0 & 0.54 \\
\hline ARB, n (\%) & 0 & $1(6)$ & 0.36 \\
\hline Diuretics, n (\%) & 0 & $1(6)$ & 0.36 \\
\hline MR-antagonist, $n(\%)$ & 0 & 0 & NA \\
\hline Colchicine, n (\%) & 0 & 0 & NA \\
\hline \multicolumn{4}{|l|}{ Medications at discharge } \\
\hline Betablocker, n (\%) & $5(17)$ & $4(25)$ & 0.7 \\
\hline ACE inhibitor, n (\%) & $12(40)$ & $4(25)$ & 0.34 \\
\hline ARB, n (\%) & $1(3)$ & 1 (6) & 1 \\
\hline Diuretics, n (\%) & 0 & $1(6)$ & 0.36 \\
\hline MR-antagonist, n (\%) & 0 & 0 & NA \\
\hline Colchicine, n (\%) & $5(17)$ & 0 & 0.14 \\
\hline
\end{tabular}

ARB, angiotensin receptor blocker; CAD, coronary artery disease; CMR, cardiac MRI; CRP, C reactive protein; LVEF, left ventricular ejection fraction; MR, mineralcorticoid receptor; NT-proBNP, NT-pro-brain natriuretic peptide.

exertion (New York Heart Association II), whereas none of the patients treated with NSAIDs reported dyspnea.

\section{Blood pressure}

Blood pressure values at the time of the initial hospital admission were similar for both groups with a mean of $123 / 75 \pm 15 / 10 \mathrm{~mm} \mathrm{Hg}$ for patients who were later treated with NSAIDs additionally and a mean of $127 / 79 \pm 16 / 13$ $\mathrm{mm} \mathrm{Hg}$ for patients later receiving standard heart failure therapy only $(\mathrm{p}=0.5$ for systolic values and $\mathrm{p}=0.18$ for diastolic values). NSAID use did not significantly change blood pressure values during follow-up with a mean blood pressure of $124 / 75 \pm 14 / 9 \mathrm{~mm} \mathrm{Hg}$ for patients having received NSAIDs and 123/75 $\pm 13 / 9 \mathrm{~mm} \mathrm{Hg}$ for patients receiving standard heart failure therapy only at 3 months $(\mathrm{p}=0.81$ and 0.83$)$ and a mean of $124 / 75 \pm 11 / 8 \mathrm{~mm} \mathrm{Hg}$ and $124 / 76 \pm 9 / 5 \mathrm{~mm} \mathrm{Hg}$ at 6 months $(\mathrm{p}=0.73$ and 0.4$)$, respectively.

However, there was a significant decrease in overall blood pressure values from initial hospital admission to hospital discharge in the general patient cohort (mean 124/76 $\pm 15 / 11 \mathrm{~mm} \mathrm{Hg}$ on admission and mean $116 / 69 \pm 12 / 10 \mathrm{~mm} \mathrm{Hg}$ at discharge, $\mathrm{p}=0.006$ and $\mathrm{p}=0.001$ ) with blood pressure values returning to baseline values during follow-up.

\section{Exercise capacity}

A total of 28 patients $(62 \%)$ underwent bicycle stress testing (standard heart failure therapy, $\mathrm{n}=12$; NSAIDs $\mathrm{n}=16$ ). There was no difference in exercise capacity between the two groups: the group receiving NSAIDs performed with $202 \pm 50 \mathrm{~W} / 9.7 \pm 2.7$ metabolic equivalents, 
100
- baseline $\sqcup 3$ months

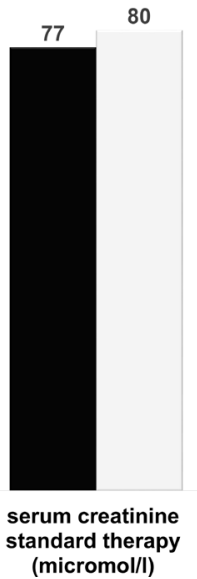

100

- baseline $\sqcup 3$ months

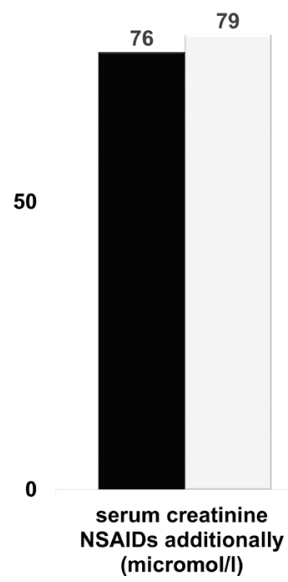

Figure 2 No significant change in serum creatinine. The left diagram indicates mean creatinine levels of patients with additional non-steroidal anti-inflammatory drugs (NSAIDs); the right diagram shows mean creatinine levels of patients with standard therapy only.

while the group with standard heart failure therapy performed with $200 \pm 67 \mathrm{~W} / 9.6 \pm 2.8$ metabolic equivalents, $p=0.87)$. One patient $(1 / 12=8 \%)$ receiving standard heart failure therapy developed increasing ventricular ectopy, syncope and convulsions during exercise stress testing. Monitoring revealed sinus tachycardia. The episode was interpreted as a seizure and was treated successfully with intravenous midazolam.

\section{8-hour Holter monitoring}

The rate of premature ventricular contractions (PVC) on 48-hour Holter monitoring was overall low among both patient populations. In patients treated with NSAIDs, mean PVC rate was $0.007 \% \pm 0.02 \%$, while it was

\section{LGE at 3 Months Post Myopericarditis}

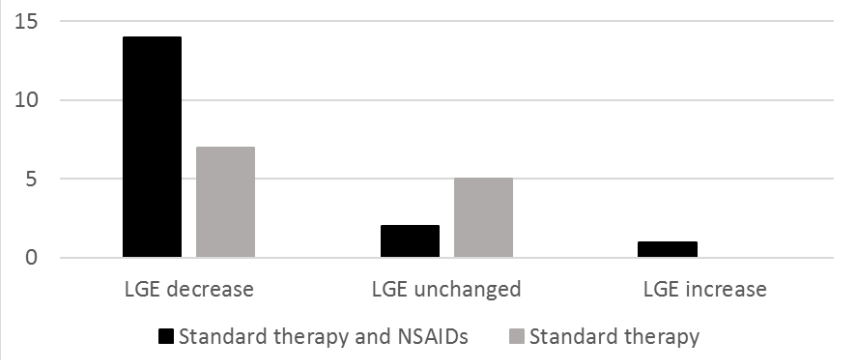

Figure 3 Trend towards improved late gadolinium enhancement (LGE) under non-steroidal anti-inflammatory drug (NSAID) therapy. In 14 of 17 patients treated with NSAIDs, there was a decreased LGE extent at 3 months compared with 7 of 12 in those with standard therapy only. LGE remained unchanged in 2 of 17 with NSAIDs and 5 of 12 without NSAIDs. One patient with NSAIDs experienced an increased LGE, while no increase of LGE was observed in those without NSAIDs.
$0.25 \% \pm 0.48 \%(\mathrm{p}=0.44)$ in the group on standard heart failure therapy.

\section{Renal function}

Based on serum levels of creatinine obtained at 3 months, there was no significant difference in kidney function between the two groups: average creatinine level in patients with NSAIDs was $76 \pm 11 \mu \mathrm{mol} / \mathrm{L}$ at baseline and $79 \pm 12 \mu \mathrm{mol} / \mathrm{L}$ at 3-month follow-up while it was $77 \pm 15 \mu \mathrm{mol} / \mathrm{L}$ at baseline and $80 \pm 15 \mu \mathrm{mol} / \mathrm{L}$ at 3 -month follow-up in patients with standard heart failure therapy $(\mathrm{p}=0.97$, figure 2$)$.

\section{CMR examination at baseline and 3-month follow-up}

All patients $(n=45)$ presented with LGE in the pericardium and myocardium at baseline. Twenty-four of those (53\%) also presented with myocardial oedema according to T2 imaging. Twelve patients $(27 \%)$ had pericardial effusion without impact on haemodynamics and three (7\%) showed pericardial thickening according to MRI imaging.

Twenty-nine patients (64\%) underwent follow-up CMR at 3 months. Among the 29 patients with CMR both at baseline and at 3-month follow-up, 17 received NSAIDs (ASA: $n=6$, ibuprofen: $n=11$ ). Eighty-two per cent of the patients (14 of 17) treated with NSAIDs experienced a decrease in LGE at 3 months as compared with $58 \%$ (7 of 12) in those not receiving NSAIDs (figure 3). However, the level of significance was not reached $(p=0.15)$. In one of the patients treated with NSAIDs, there was an increase in LGE at 3 months-no increase in LGE was observed in the group without NSAIDs. LGE resolved entirely in three patients (NSAID group: $n=2$, standard therapy group: $\mathrm{n}=1, \mathrm{p}=0.77)$. At 3 -month follow-up, oedema was still present in one patient $(1 / 29 ; 3 \%)$, five patients $(17 \%)$ continued to have signs of pericardial effusion, while one patient with pericardial effusion continued to have pericardial thickening.

\section{Adverse events during follow-up}

There was one adverse event $(6 \%)$ in the group with standard heart failure therapy: One of the patients experienced polymorphic ventricular tachycardia with cardiac arrest at 6-month follow-up, requiring resuscitation and implantation of a cardioverter defibrillator. This patient initially presented with an almost normal LVEF of $50 \%$ but had previously been diagnosed with LGE persistence on CMR at 3-month follow-up. One adverse event $(3 \%)$ occurred in the group with NSAIDs. The patient experienced an asymptomatic episode of non-sustained ventricular tachycardia on 48-hour Holter at 3-month follow-up, which lasted for 9 beats. In this case, the initial LVEF was $56 \%$ and follow-up MRI showed a decrease in LGE.

\section{DISCUSSION}

This is the first case-control study to evaluate the effect of NSAIDs on the clinical course of myopericarditis on 
top of standard heart failure therapy. The results demonstrate that NSAIDs are safe over an observation period of 12 months both clinically as well as regarding blood pressure, renal function, arrhythmias and exercise tolerance in such patients. Furthermore, CMR suggests that NSAIDs may even be protective and reduce LGE, an index of myocardial damage, although significance was not reached due to low numbers.

In a previous study by Buiatti and colleagues, ${ }^{22} 62$ patients were diagnosed with myopericarditis based on clinical presentation, laboratory parameters, ECG and echocardiography. Similar to our case-control study, among 61 patients treated with NSAIDs, no cardiovascular adverse events were reported after 1 year of therapy. However, no control group was provided in this study. In a multicentre study by Imazio and colleagues, ${ }^{23}$ myocardial involvement was determined by CMR at baseline. In an overall patient population of 486 cases with pericarditis, there were 140 patients with myocardial involvement. Out of these, $76 \%$ or 106 patients were treated with NSAIDs with ASA being the predominant drug. For treatment of pericarditis, a dose of 750-1000 mg orally every 6 or 8 hours for 7-10 days was chosen with gradual decrease of the dose over 2-3 weeks until treatment was stopped. However, patients with myocardial involvement were given a lower dosage of $500 \mathrm{mg}$ every 8-12 hours, which is somewhat less than the dosage generally used for anti-inflammatory effects in pericardial disease. ${ }^{10}$ After a median of 36 months, no deaths or heart failure were recorded.

Very recently, Ammirati and colleagues published data from a retrospective multicentre registry examining characteristics, in-hospital treatment and long-term outcome of patients with acute myocarditis. ${ }^{30}$ The final study population included 443 patients. NSAIDs were used in 267 out of 433 patients (44\% NSAIDs in cases with complicated myocarditis and $67.6 \%$ NSAIDs in uncomplicated myocarditis) suggesting safety for this drug class. CMR at baseline and at follow-up was obtained; however, time course of LGE was not examined. Main finding of this study was that adverse events after acute myocarditis were lower than previously reported and mainly driven by reduced LVEF, ventricular arrhythmias or low cardiac output at presentation. However, Ammirati and colleagues suggested safety of NSAIDs and findings of this registry did not support the findings of NSAIDs leading to worse prognosis in viral myocarditis as observed in murine models.

Thus, although adverse effects of NSAIDs have been observed in animal studies in the setting of experimental myocarditis, our findings support prior, but less well-controlled studies in humans, which found no major adverse cardiovascular events under NSAID therapy during longterm follow-up in myopericarditis. Follow-up in these prior studies included a clinical examination, blood examinations, echocardiography ${ }^{22}$ and exercise treadmill testing. ${ }^{23}$ In one recent study in patients with acute myocarditis receiving NSAID therapy, CMR follow-up was included but time course of LGE was not examined. Our case-control study substantiates these preliminary findings by others and provides additional information with CMR demonstrating a trend for a reduction in LGE at 3 months in those receiving NSAIDs.

\section{CONCLUSION}

Our data suggest a notion of safety for NSAIDs in myopericarditis and preserved LVEF. We conclude that further evaluation of NSAIDs in patients with myopericarditis in a prospective double-blinded randomised trial should be considered.

\section{LIMITATIONS}

Given the small sample size, significance levels of LGE dynamic may vary in a larger patient cohort. Nine patients among the standard therapy group had to be excluded from analysis to create two groups with homogenous baseline conditions in this matched case-control study. Excluded patients were older and had worse LVEF. Five patients were lost to follow-up.

Acknowledgements The authors would like to thank the clinical staff and nurses of the Division of Cardiology at the University Hospital Zurich for their help with patient enrolment. Also, they would like to thank the Swiss National Science Foundation, the University of Zurich, the Holcim Foundation and the Walter and Gertrud Siegenthaler Stiftung for financial support of this project.

Contributors All authors contributed in either clinical follow-up on patients or giving valuable input in order to improve the manuscript.

Funding Project Grant of the Swiss National Science Foundation, Forschungskredit of the University of Zurich FK-17-047; the Holcim Foundation, Walter and Gertrud Siegenthaler Stiftung at the University of Zurich.

Competing interests None declared.

Patient consent for publication Obtained.

Ethics approval Ethics committee of the Kanton of Zurich.

Provenance and peer review Not commissioned; externally peer reviewed.

Data availability statement Data are available upon reasonable request.

Open access This is an open access article distributed in accordance with the Creative Commons Attribution Non Commercial (CC BY-NC 4.0) license, which permits others to distribute, remix, adapt, build upon this work non-commercially, and license their derivative works on different terms, provided the original work is properly cited, appropriate credit is given, any changes made indicated, and the use is non-commercial. See: http://creativecommons.org/licenses/by-nc/4.0/.

\section{REFERENCES}

1. Heidecker B, Kittleson MM, Kasper EK, et al. Transcriptomic biomarkers for the accurate diagnosis of myocarditis. Circulation 2011;123:1174-84.

2. Sagar S, Liu PP, Cooper LT. Myocarditis. The Lancet 2012;379:738-47.

3. Heymans S, Eriksson U, Lehtonen J, et al. The Quest for New Approaches in Myocarditis and Inflammatory Cardiomyopathy. J Am Coll Cardiol 2016;68:2348-64.

4. Caforio ALP, Pankuweit S, Arbustini E, et al. Current state of knowledge on aetiology, diagnosis, management, and therapy of myocarditis: a position statement of the European Society of cardiology Working Group on myocardial and pericardial diseases. Eur Heart J 2013;34:2636-48.

5. Cooper LT. Myocarditis. N Engl J Med 2009;360:1526-38.

6. Schultheiss H-P, Kühl U, Cooper LT. The management of myocarditis. Eur Heart J 2011;32:2616-25.

7. Frustaci A, Chimenti C, Calabrese F, et al. Immunosuppressive therapy for active lymphocytic myocarditis: virological and 
immunologic profile of responders versus nonresponders. Circulation 2003;107:857-63.

8. Cooper LT, Hare JM, Tazelaar HD, et al. Usefulness of immunosuppression for giant cell myocarditis. Am J Cardiol 2008;102:1535-9.

9. Montero S, Aissaoui N, Tadié J-M, et al. Fulminant giant-cell myocarditis on mechanical circulatory support: management and outcomes of a French multicentre cohort. Int $J$ Cardiol 2018;253:105-12.

10. Adler Y, Charron P, Imazio M, et al. 2015 ESC Guidelines for the diagnosis and management of pericardial diseases: The Task Force for the Diagnosis and Management of Pericardial Diseases of the European Society of Cardiology (ESC)Endorsed by: The European Association for Cardio-Thoracic Surgery (EACTS). Eur Heart $J$ 2015;36:2921-64.

11. Costanzo-Nordin MR, Reap EA, O'Connell JB, et al. A nonsteroid anti-inflammatory drug exacerbates Coxsackie B3 murine myocarditis. J Am Coll Cardiol 1985;6:1078-82.

12. Rezkalla S, Khatib G, Khatib R. Coxsackievirus B3 murine myocarditis: deleterious effects of nonsteroidal anti-inflammatory agents. J Lab Clin Med 1986;107:393-5.

13. Khatib R, Reyes MP, Smith F, et al. Enhancement of coxsackievirus B4 virulence by indomethacin. J Lab Clin Med 1990;116:116-20.

14. Khatib R, Reyes MP, Khatib G, et al. Focal ventricular thinning caused by indomethacin in the late phase of coxsackievirus B4 murine myocarditis. Am J Med Sci 1992;303:95-8.

15. Ruschitzka F, Borer JS, Krum H, et al. Differential blood pressure effects of ibuprofen, naproxen, and celecoxib in patients with arthritis: the PRECISION-ABPM (prospective randomized evaluation of celecoxib integrated safety versus ibuprofen or naproxen ambulatory blood pressure measurement) trial. Eur Heart $J$ 2017;38:3282-92.

16. Schneider V, Lévesque LE, Zhang B, et al. Association of selective and conventional nonsteroidal antiinflammatory drugs with acute renal failure: a population-based, nested case-control analysis. Am J Epidemiol 2006;164:881-9.

17. Lurz P, Luecke C, Eitel I, et al. Comprehensive cardiac magnetic resonance imaging in patients with suspected myocarditis: the MyoRacer-Trial. J Am Coll Cardiol 2016;67:1800-11.

18. Kumar A, Sato K, Yzeiraj E, et al. Quantitative Pericardial Delayed Hyperenhancement Informs Clinical Course in Recurrent Pericarditis. JACC Cardiovasc Imaging 2017;10:1337-46.
19. Patriki D, Gresser E, Manka R, et al. Approximation of the Incidence of Myocarditis by Systematic Screening With Cardiac Magnetic Resonance Imaging. JACC Heart Fail 2018;6:573-9.

20. Sudano I, Flammer AJ, Périat D, et al. Acetaminophen increases blood pressure in patients with coronary artery disease. Circulation 2010;122:1789-96.

21. Kato H, Fujigaki Y, Inoue R, et al. Therapeutic dose of acetaminophen as a possible risk factor for acute kidney injury: learning from two healthy young adult cases. Intern Med 2014;53:1531-4.

22. Buiatti A, Merlo M, Pinamonti B, et al. Clinical presentation and long-term follow-up of perimyocarditis. J Cardiovasc Med 2013;14:235-41.

23. Imazio M, Brucato A, Barbieri A, et al. Good prognosis for pericarditis with and without myocardial involvement: results from a multicenter, prospective cohort study. Circulation 2013;128:42-9.

24. Imazio M, Brucato $A$, Maestroni S, et al. Risk of constrictive pericarditis after acute pericarditis. Circulation 2011;124:1270-5.

25. Murashita T, Schaff HV, Daly RC, et al. Experience with pericardiectomy for constrictive pericarditis over eight decades. Ann Thorac Surg 2017;104:742-50.

26. Schumm J, Greulich S, Wagner A, et al. Cardiovascular magnetic resonance risk stratification in patients with clinically suspected myocarditis. J Cardiovasc Magn Reson 2014;16.

27. Aquaro GD, Perfetti M, Camastra G, et al. Cardiac MR With Late Gadolinium Enhancement in Acute Myocarditis With Preserved Systolic Function: ITAMY Study. J Am Coll Cardiol 2017;70:1977-87.

28. Ganesan AN, Gunton J, Nucifora G, et al. Impact of late gadolinium enhancement on mortality, sudden death and major adverse cardiovascular events in ischemic and nonischemic cardiomyopathy: a systematic review and meta-analysis. Int $J$ Cardiol 2018;254:230-7.

29. Berg J, Kottwitz J, Baltensperger N, et al. Cardiac magnetic resonance imaging in myocarditis reveals persistent disease activity despite normalization of cardiac enzymes and inflammatory parameters at 3-month follow-up. Circ Heart Fail 2017;10.

30. Ammirati E, Cipriani M, Moro C, et al. Clinical presentation and outcome in a contemporary cohort of patients with acute myocarditis. Circulation 2018;138:1088-99. 